\title{
FO Hydrophone with Hydrostatic Pressure Compensation: comparative experiment with a conventional piezo hydrophone
}

\author{
L.K. Cheng and D. de Bruijn \\ TNO Institute of Applied Physics (TNO/TPD) \\ PO Box 155, 2600 AD Delft, The Netherlands \\ Tel: +31 15 2692000, Fax: +31 152692111 \\ E-mail: cheng@tpd.tno.nl
}

\section{Introduction}

This paper presents the performance of a TPD FO hydrophone with Hydrostatic Pressure Compensation. In particularly a comparison with a conventional piezo hydrophone system is discussed.

\section{Sensor design}

The TPD hydrophone is based on a flexural disc [1]. In the disc type of FO hydrophone, the sensing leg of a FO interferometer is coiled and fixed firmly on the disc. Pressure sensing by the disc type of FO hydrophone is based on the bending induced surface strains of the disc due to uniform pressure. This simple design can be easily extended to a push pull system and/or an acceleration canceling system [2].

\section{Sensitivity of the disk type of FO hydrophone}

In a FO hydrophone, the normalized sensitivity $S_{n}$ is defined as the ratio of the strain $\varepsilon$ in the sensing fiber and the applied pressure $\mathrm{p}$ :

$$
S_{n}=0.78 \frac{\varepsilon}{p}
$$

where the factor 0.78 is a result of the photoelastic effect of strain in the fiber. For the disc type of FO hydrophone, $S_{n}$ depends on the flexibility of the disc while the absolute sensitivity $S_{a}$ is proportional to the product of $S_{n}$ and the length $L_{s}$ of the sensing fiber. An higher absolute sensitivity can be achieved by either an higher normalized sensitivity or a longer sensing fiber.

The length $L_{s}$ of the sensing fiber in a fiber coil with inner radius $b$, outer radius $c$ and a fiber diameter $\mathrm{D}$ can be calculated as:

$$
L_{s}=\frac{\pi}{D}\left(c^{2}-b^{2}\right)
$$

The fiber diameter $\mathrm{D}$ and the inner radius $\mathrm{b}$ are determined by the properties of the commercially available fiber. So, the length of the sensing fiber is determined by the outer radius $c$ of the coil. In the disc type of FO hydrophone, the outer radius of the coil is restricted by the radius of the disc. Since a large size of the FO hydrophone is usually undesirable, the length of the sensing fiber is limited. (For example, for $c=15 \mathrm{~mm}, \mathrm{~b}=5 \mathrm{~mm}$ and $\mathrm{D}=135 \mu \mathrm{m}, \mathrm{L}_{\mathrm{s}}$ is calculated to be about $4.6 \mathrm{~m}$ ) 
Provided the length of the sensing fiber is limited, the absolute sensitivity in a disc type of FO hydrophone can only be increased by an higher normalized sensitivity. However, an higher normalized sensitivity will limit the operation pressure.

\section{Limited operation pressure}

In the conventional FO hydrophone designs, the sensor is not able to distinguish the acoustic pressure from the hydrostatic pressure which is caused by the operation depth. The problem of a limited operation pressure is caused by the fact that optical fiber has a limited maximum strain of about 1\%. Regarding Eq. (1), the maximum operation pressure of the FO hydrophone has to be lower than the pressure $\mathrm{P}_{1 \%}$ which corresponds to a strain of $1 \%$ in the sensing fiber $\left(\mathrm{P}_{1 \%}=\right.$ $\left.0.0078 / S_{n}\right)$. Consequently, a FO hydrophone with an higher $S_{n}$ will reach the $1 \%$ strain at a lower pressure. In Fig. 1 the relation between $P_{1 \%}$ and $S_{n}$ is shown. For a certain level of maximum operation pressure, the normalized sensitivity is limited.

\section{FO hydrophone with Hydrostatic Pressure Compensation}

To solve the problem of a limited sensitivity due to a desired maximum operation pressure, we invented an Hydrostatic Pressure Compensation (HPC) mechanism. This mechanism is implemented in among others our M8c FO hydrophone. The M8c has an high normalized sensitivity of about $-280 \mathrm{~dB}$ re $1 / \mu \mathrm{Pa}$ [3]. The HPC is found to have no noticeable influence on the normalized sensitivity while the maximum operation pressure is increased from $0.7 \mathrm{MPa}$ to more than $3.5 \mathrm{MPa}$. The normalized sensitivity of M8c is measured as a function of the hydrostatic pressure and shown in Fig. 2.

\section{Comparative experiment between a FO hydrophone with HPC and a conventional piezo hydrophone system}

In 1997 we performed a comparative experiment between one of our FO hydrophones with HPC and a conventional piezo hydrophone. The response the two systems for a pressure change with an high amplitude is recorded. During the measurement both hydrophones are put in a pressure tank and a $200 \mathrm{~Hz}$ acoustic signal is applied to the hydrophones (Fig. 3). The output signal of both systems are displayed in Fig. 4.

At the beginning of the experiment, the pressure in the tank is set at $2.5 \mathrm{MPa}$. The $200 \mathrm{~Hz}$ acoustic signal is detected by both systems. At $\mathrm{t}=\mathrm{T} 0$, we opened the valve of the pressure tank, water flowed out of the pressure tank and the pressure in the tank declined. At $t=T 1$, when the pressure is dropped to about $0.8 \mathrm{MPa}$, the valve is closed. Between $\mathrm{T} 0$ and $\mathrm{T} 1$ the pressure is assumed to be decreasing gradually while at T0 and T1 there was a sudden change in the pressure.

Regarding the piezo hydrophone system, the sudden change in the pressure at $\mathrm{T} 0$ and $\mathrm{T} 1 \mathrm{results}$ in an abrupt change in the output signal. During the pressure change from $2.5 \mathrm{MPa}$ to $0.8 \mathrm{MPa}$ between T0 and T1 (about 5 seconds), the piezo hydrophone system was not able to detect the $200 \mathrm{~Hz}$ acoustic signal. More important is that even a few seconds after the sudden pressure change at $\mathrm{T} 1$, the piezo hydrophone system was still not capable to detect the $200 \mathrm{~Hz}$ acoustic signal. It took up to $\mathrm{t}=\mathrm{T} 2$ (T2-T1 = about 7 seconds) before the piezo system was fully recovered.

The pressure change from $2.5 \mathrm{MPa}$ to $0.8 \mathrm{MPa}$ between $\mathrm{T} 1$ and $\mathrm{T} 0$ has no visible influence on the $200 \mathrm{~Hz}$ output signal of the TPD FO hydrophone system. Even the sudden change in the pressure at T0 and T1 did not have any noticeable effect. The $200 \mathrm{~Hz}$ acoustic signal is found to be detected during the whole experiment. This advantage of the TPD FO hydrophone in comparison to the conventional piezo hydrophone system could be of overriding importance in critical situations. Detailed investigation of this effect is planned in early 1998. 


\section{Acknowledgement}

The authors would like to acknowledge the Royal Netherlands Navy who funded the FO hydrophone research.

\section{References}

1. Brown D.A., et al., "Fiber Optic Flexural Disk Microphone", Proceedings of SPIE, Vol. 985, pp. 172, 1988.

2. Brown D.A., et al., "High-Sensitivity, Fiber-Optic, Flexural Disk Hydrophone with Reduced Acceleration Response", Fiber and Integrated Optics, Vol. 8, pp. 169, 1989.

3. Cheng L.K. and de Bruijn D., "Fieldtest of a Fiber Optic Hydrophone", $11^{\text {th }}$ International Conference on Optical Fiber Sensors, pp. 184, 1996.

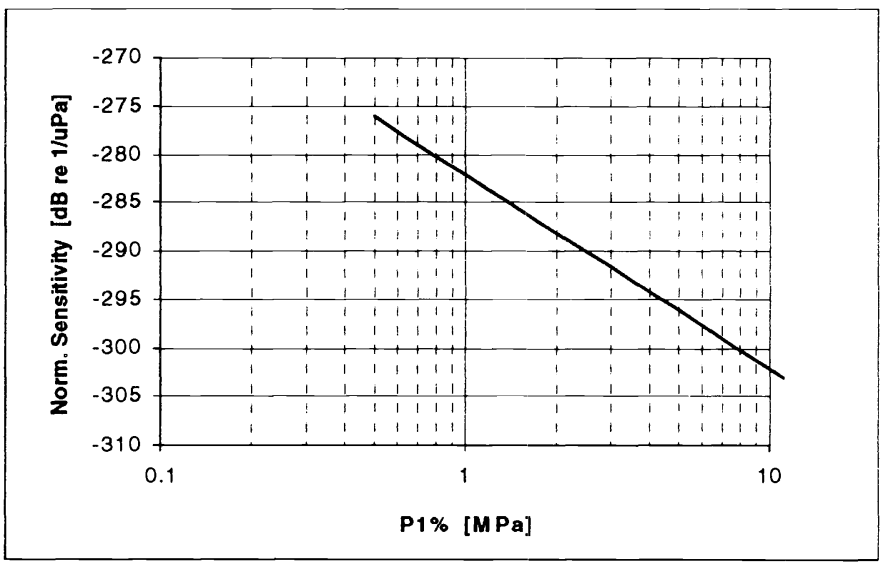

Figure 1 Relation between the norm. sensitivity and the pressure which corresponds to a strain of $1 \%$ in the optical fiber.

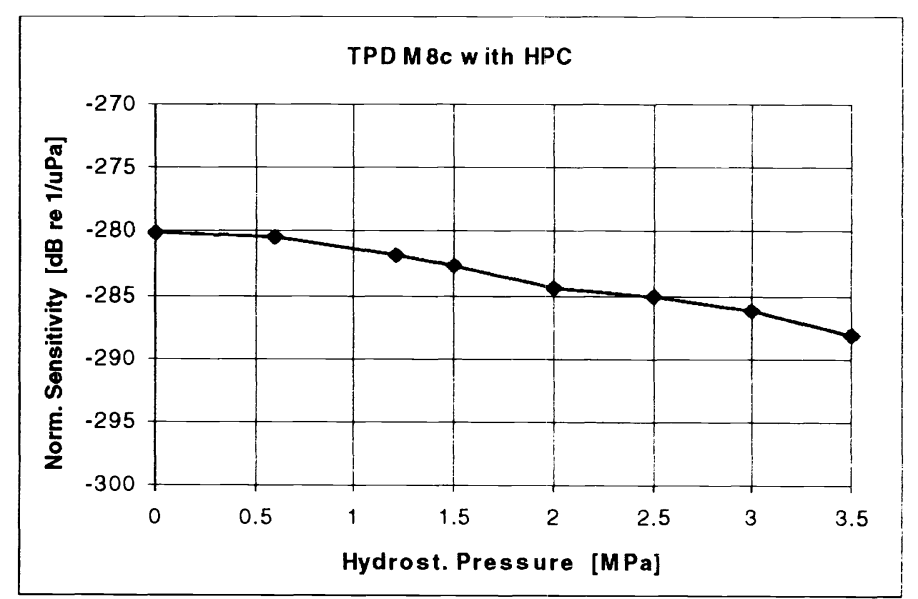

Figure 2 The norm. sensitivity of the M8c with HPC as a functie op the hydrostatic pressure. 


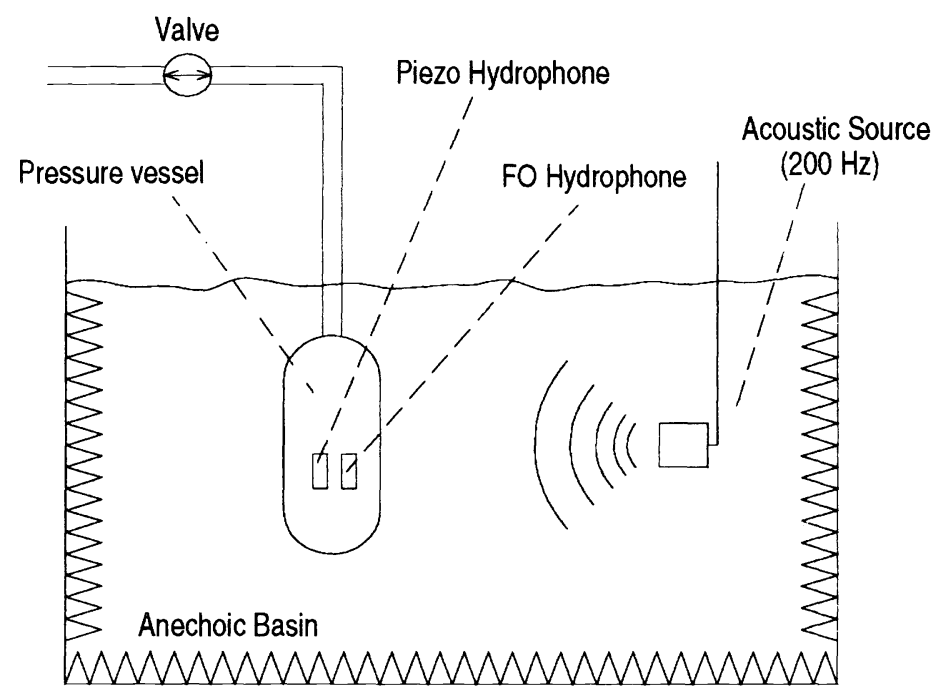

Figure 3 Experimental configuration.
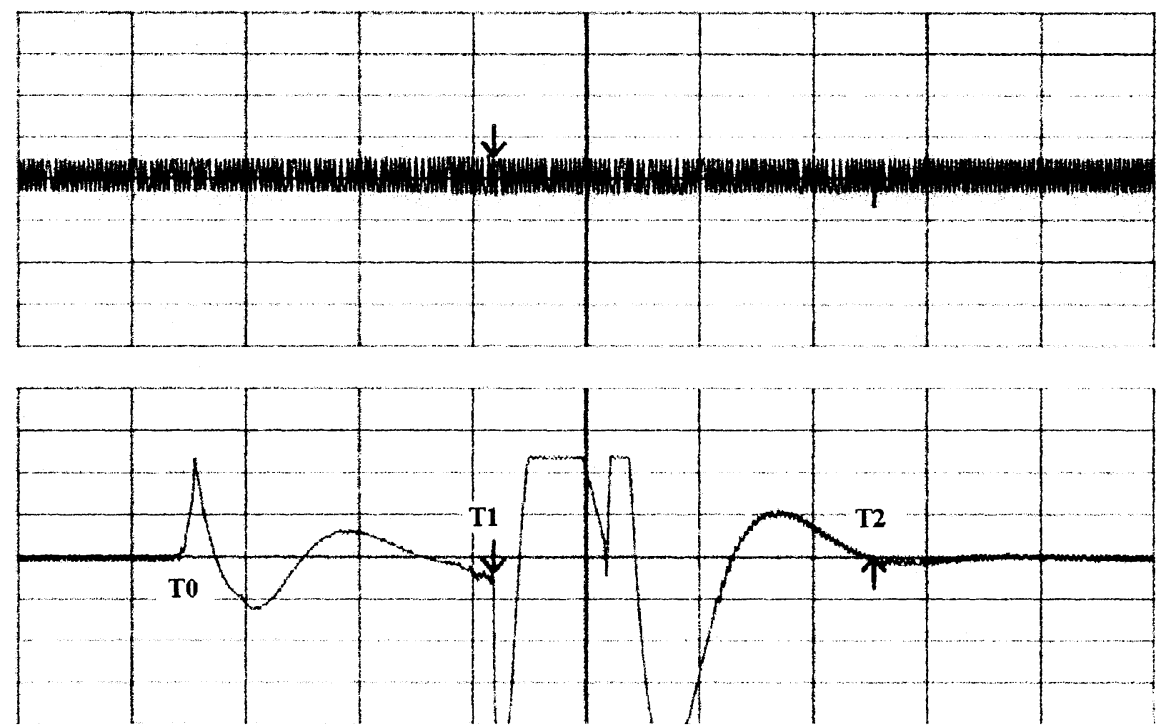

Figure 4 Influence of rapid change in pressure on the operation of the TPD FO hydrophone with HPC (upper figure) and a conventional piezo hydrophone (lower figure). During the experiment, a $200 \mathrm{~Hz}$ acoustic signal is applied to the two sensors The scale of the time-base is 2 seconds per division and the scale of the vertical axis is 1 Volt per division. 